\title{
CUIDADO EDUCATIVO COM DIABÉTICOS INSULINODEPENDENTES: POSSIBILIDADES TERAPÊUTICAS NA ATENÇÃO PRIMÁRIA EM SAÚDE
}

\footnotetext{
CARDOSO, E. L. S. ${ }^{1}$; TAVARES, B. L. O. ${ }^{2}$; BARROS, I. N. M. ${ }^{3}$; SANTOS, E. F. A. ${ }^{4}$ \& PONTES, A. R. B. ${ }^{5}$

${ }^{1}$ Bolsista e graduando em Enfermagem pela Universidade Federal do Pará (UFPA). E-mail: ebersonluan@ gmail.com; ${ }^{2}$ Bolsista e graduanda em Enfermagem pela Universidade Federal do Pará (UFPA). E-mail: brunalotavares@ hotmail.com; ${ }^{3}$ Voluntária e graduanda em Enfermagem pela Universidade Federal do Pará (UFPA). E-mail: ingridnicollebarros@gmail.com; ${ }^{4}$ Enfermeira Assistencial, Especialista em Regulação em Saúde, Unidade Municipal de Saúde do Guamá, Secretaria Municipal de Saúde de Belém - PA. E-mail: edenilzasantos66@yahoo.com.br; ${ }^{5}$ Enfermeira, Doutora em Patologia de Doenças Tropicais, Docente Adjunta da Faculdade de Enfermagem da Universidade Federal do Pará, Coordenadora do Projeto de Extensão. E-mail: anapontes@ufpa.br.

DOI: https://doi.org/10.32356/exta.v2.n18.33928 - Artigo submetido em 15/10/2018
}

\section{RESUMO}

Este estudo visa descrever as experiências extensionistas de acadêmicos de Enfermagem na promoção do autocuidado e prevenção de agravos em usuários diabéticos insulinodependentes, através da utilização de estratégias educativas em saúde em grupo e individuais, visando a realização da técnica correta de auto-aplicação da insulina e o controle glicêmico adequado. Dessa maneira, as estratégias metodológicas utilizadas foram: ações de cuidado educativo em grupo, através da abordagem de temáticas voltadas para o autocuidado em diabetes, em especial autoadministração de insulina, realizadas com o auxílio de tecnologias leve-duras em saúde; e consultas de enfermagem individuais para educação em saúde dos usuários, com o enfoque em enfrentamento das principais dificuldades na aderência à terapia insulínica e capacitação para autoadministração da insulina subcutânea. O desenvolvimento das atividades proporcionou maiores conhecimentos sobre a realidade dos usuários insulinodependentes, a percepção das dificuldades frente à terapêutica, bem como reafirmou a importância da assistência de enfermagem, com ênfase no autocuidado, como uma alternativa encontrada para viabilizar a adesão ao tratamento, melhorar a qualidade de vida e reduzir os elevados encargos à família, à sociedade e ao sistema público de saúde. A vivência destacou ainda a educação terapêutica como sendo essencial na atenção primária para informar, motivar e fortalecer a pessoa e a família, cabendo aos profissionais de saúde, especialmente os enfermeiros, a adoção de atividades educativas como reforço aos programas de atenção integral em saúde.

PALAVRAS-CHAVE: Educação em Saúde. Diabetes Mellitus. Assistência de Enfermagem.

EDUCATIONAL CARE WITH INSULINODEPENT DIABETICS: THERAPEUTIC POSSIBILITIES IN PRIMARY HEALTH CARE

\section{ABSTRACT}

This study aims to describe extensionist experiences of nursing students in the promotion of self-care and prevention of diseases in insulin-dependent diabetic patients, through the use of group and individual health education strategies, aiming at the correct technique of self-application of insulin and adequate glycemic control. In this way, the methodological strategies used were: group educational care actions, through the approach of the themes focused on selfcare in diabetes, in particular self-administration of insulin, performed with the help of mild-to-health technologies; and individual nursing consultations for health education of the users, with a focus on coping with the main difficulties in adherence to insulin therapy and training in self-administration of subcutaneous insulin. The development of activities provided greater insight into the reality of insulindependent users, the perception of difficulties with therapy, and reaffirmed the importance of nursing care, with emphasis on self-care, as an alternative found to enable adherence to treatment, quality of life and reduce the high burden on the family, society and the public health system. The experience also highlighted therapeutic education as essential in primary care to inform, motivate and strengthen the person and the family, and it is up to health professionals, especially nurses, to adopt educational activities to reinforce comprehensive health care programs.

KEYWORDS: Health Education. Diabetes Mellitus. Nursing Care 


\section{INTRODUÇÃO}

O diabetes mellitus (DM) é uma doença de características progressivas e crônicas que requer contínua atenção e acompanhamento da equipe multiprofissional de saúde, bem como o investimento em programas eficazes e viáveis com enfoque em estratégias educativas em saúde, visando diminuir as complicações crônicas, estimular a aderência ao tratamento e aumentar a qualidade de vida dos usuários que convivem com a doença (GUIDONI et al., 2009).

Devido a possibilidade de levar a complicações agudas e crônicas, o diabetes mellitus requer uma terapia onde sejam empregados antidiabéticos orais, bem como insulina, rotina em casos de DM tipo 1 e recentemente utilizada no DM tipo 2 (SBEM; ABRAN, 2011). Para tanto, a diminuição das chances de complicações e controle da glicemia é alcançada com diárias doses de insulina (STACCIARINI; PACE; HAAS, 2009).

A lei federal $\mathrm{n}^{\circ} 11.347$ assegura a distribuição gratuita de materiais utilizados tanto na auto aplicação de insulina, quanto no controle de glicemia, por entidades do governo desde 2006, mas só isso não basta para o sucesso do tratamento e prevenção de agravos. Usuários abandonam o tratamento por muitas vezes não saberem lidar com procedimentos invasivos, não saberem empregar técnicas corretas de administração do medicamento e desconhecerem os múltiplos locais alvos de aplicação. São nestas lacunas que atividades educativas agem, pois estimulam o sujeito a trabalhar como autônomo e responsável de seu estado de saúde através do conhecimento (BARROS; SOUZA, 2011).

Com o intuito de intervir na realidade de usuários diabéticos que realizam o tratamento em domicílio, o projeto de extensão "Promoção de saberes sobre a insulinoterapia subcutânea aos pacientes diabéticos da UBS do Guamá", da Pró-Reitoria de Extensão da Universidade Federal do Pará (PROEX-UFPA), do qual este trabalho é fruto, desenvolve na comunidade atividades de educação em saúde e proporciona espaços de discussão, especialmente aos que são atendidos na atenção primária em saúde. O planejamento das atividades se deu a partir de conhecimento prévio da realidade a qual os usuários viviam, com destaque para suas dúvidas e angústias com posterior elaboração de medidas interventivas.

Partindo disso, o objetivo do presente estudo é descrever a experiência de discentes do curso de Enfermagem na promoção do autocuidado e prevenção de agravos de usuários diabéticos insulinodependentes, através da utilização de estratégias educativas em saúde em grupo e individual. 


\section{EDUCAÇÃO EM SAÚDE EM DIABETES}

A educação em saúde voltada para o diabetes, por esta ser uma doença de complexo tratamento, requer adoção de abordagens inovadoras, fugindo do padrão tradicional centrado na doença, onde os usuários acabam se tornando meros depósitos dos conhecimentos dos profissionais de saúde. Para que este cenário mude devem ser evidenciados o estímulo ao desenvolvimento de habilidades e protagonismo da sua própria saúde, bem como o pensamento crítico e tomada consciente de decisões (CYRINO; SCHRAIBER; TEIXEIRA, 2009).

Dessa maneira, para que as intervenções junto aos usuários com diabetes sejam verdadeiramente educativas, estas devem olhar o indivíduo como ser social, problematizando o cotidiano do mesmo e valorizando as experiências que estes trazem, assim como as diferentes realidades onde estão inseridos. Além do exposto, é essencial considerar o nível de habilidade do usuário para se ajudar, bem como o contexto familiar e comunitário, fatores esses que influenciam diretamente nas práticas de autocuidado requeridas (COSTA, 2014).

No que se refere às práticas assistenciais na atenção primária em saúde, o enfermeiro se destaca como um profissional fundamental da equipe, lançando mão das estratégias de educação em saúde, individuais e coletivas, como atividades inerentes à sua atuação. Dentre as ferramentas utilizadas privativamente pelos enfermeiros para cuidado educativo individual em saúde, além de cuidados específicos, destacamos a consulta de enfermagem, por meio da qual é possível a identificação das respostas do indivíduo, família e comunidade, garantindo a implementação de cuidados individualizados e uma assistência de qualidade, visando a promoção, prevenção, recuperação e reabilitação da saúde do indivíduo com diabetes (COFEN, 2009).

A possibilidade de trabalho com grupos de usuários na atenção primária também é uma alternativa para as práticas assistenciais da equipe de saúde, permitindo a valorização e troca dos diversos saberes e a possibilidade de intervir criativamente no processo saúde-doença de cada pessoa, bem como auxiliar na promoção da compreensão de situações enfrentadas pelo indivíduo (DIAS; SILVEIRA; WITT, 2009). Se faz imprescindível, portanto, que os profissionais da saúde discutam e aprendam como proceder o trabalho com grupos, uma vez que esta modalidade de atenção coletiva está sendo adotada com frequência como prática educativa e preventiva (MENEZES; AVELINO, 2016). 


\section{MATERIAIS E MÉTODOS}

Trata-se de um estudo transversal, exploratório, de abordagem qualitativa, do tipo relato de experiências, desenvolvido no decorrer das atividades extensionistas do projeto anteriormente citado, durante os meses de agosto de 2017 a julho de 2018. O projeto, em sua primeira versão, conta com uma equipe formada por duas enfermeiras docentes e uma assistencial, além de bolsistas e voluntários do curso de graduação em enfermagem.'

As atividades foram direcionadas e realizadas junto aos usuários diabéticos do tipo 2 que utilizavam a insulinoterapia como terapia complementar para controle do diabetes, inseridos no Sistema de Cadastramento e Acompanhamento de Diabéticos e Hipertensos (HIPERDIA) de uma unidade básica de saúde do município de Belém - PA, sendo contemplados, até o mês de julho/2018, um quantitativo de 42 usuários. Os encontros aconteciam em salas reservadas no Laboratório de Habilidades Humanas (LHH/UFPA), anexo da Unidade de Saúde do Guamá, Belém, Pará, em caráter quinzenal, após os convites para os encontros terem sido efetuados antecipadamente via ligação telefônica.

As estratégias metodológicas utilizadas foram:

a) Ações de cuidado educativo em grupo, através da abordagem de temáticas voltadas para o autocuidado em diabetes, em especial autoadministração de insulina, realizadas com o auxílio de tecnologias leve-duras em saúde; e

b) Consultas de enfermagem individuais para educação em saúde individual dos usuários, com o enfoque no enfrentamento das principais dificuldades na adesão à terapia insulínica e na capacitação para autoadministração da insulina subcutânea em domicílio.

As experiências foram registradas por meio de observação e anotações feitas pelos acadêmicos, responsáveis pela condução dos encontros, dinâmicas, tecnologias educativas e da avaliação do entendimento dos usuários.

Os materiais utilizados para a realização das ações foram inteiramente de autoria e confecção dos acadêmicos (com exceção dos insumos), sendo estes moldados para contemplar temáticas indispensáveis do DM e da terapêutica insulínica, dentre as quais podemos citar: $\mathrm{O}$ que é diabetes?; Sinais e sintomas do diabetes; Como prevenir agravos relacionados ao diabetes; Adesão ao tratamento; Insulinoterapia (técnica de administração subcutânea, principais áreas de aplicação, importância do rodízio terapêutico e descarte adequado dos insumos) e verificação glicêmica. Dentre os materiais produzidos podemos citar: 
a) Flipcharts, enfatizando os principais conceitos a partir de palavras e imagens chaves direcionadas para o tratamento, utilizado para deter a atenção dos usuários, facilitar a exposição da temática e auxiliar no processo avaliativo ao final das ações (Figura 1);

FIGURA 1 - Flipcharts utilizados para auxílio da exposição das temáticas

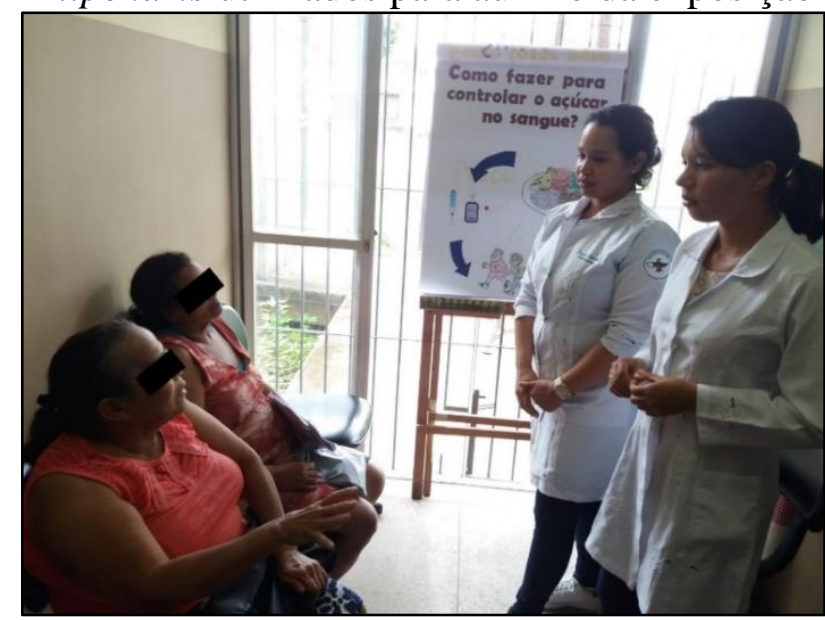

FONTE: Os autores (2018)

b) Jogo de Mitos \& Verdades sobre a insulinoterapia, com a utilização de placasrespostas, por meio do qual era oportunizado aos usuários a desmistificação de crenças sobre a doença e a terapêutica, bem como manifestação de suas dúvidas. Representava também um momento de avaliação do método empregado (Figura 2);

FIGURA 2 - Placas-respostas utilizadas no jogo Mitos \& Verdades sobre a insulinoterapia

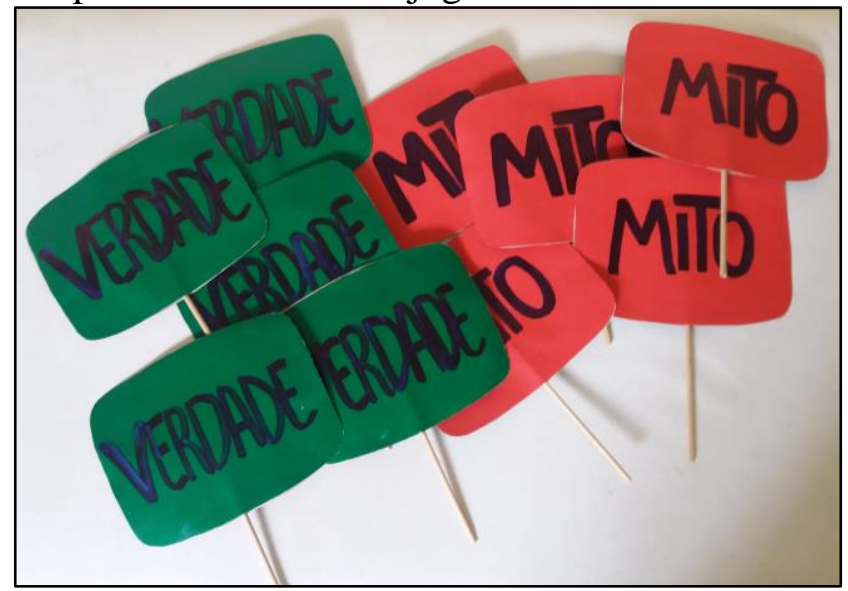

FONTE: Os autores (2018)

c) Boneco de pano, tecnologia em saúde desenvolvida para evidenciar as principais áreas do corpo onde é possível proceder a técnica da autoadministração de insulina 
subcutânea, sendo este de grande relevância no momento prático ofertado aos usuários (Figura 3).

FIGURA 3 - Boneco de pano para demonstração de áreas de auto-aplicação da insulina

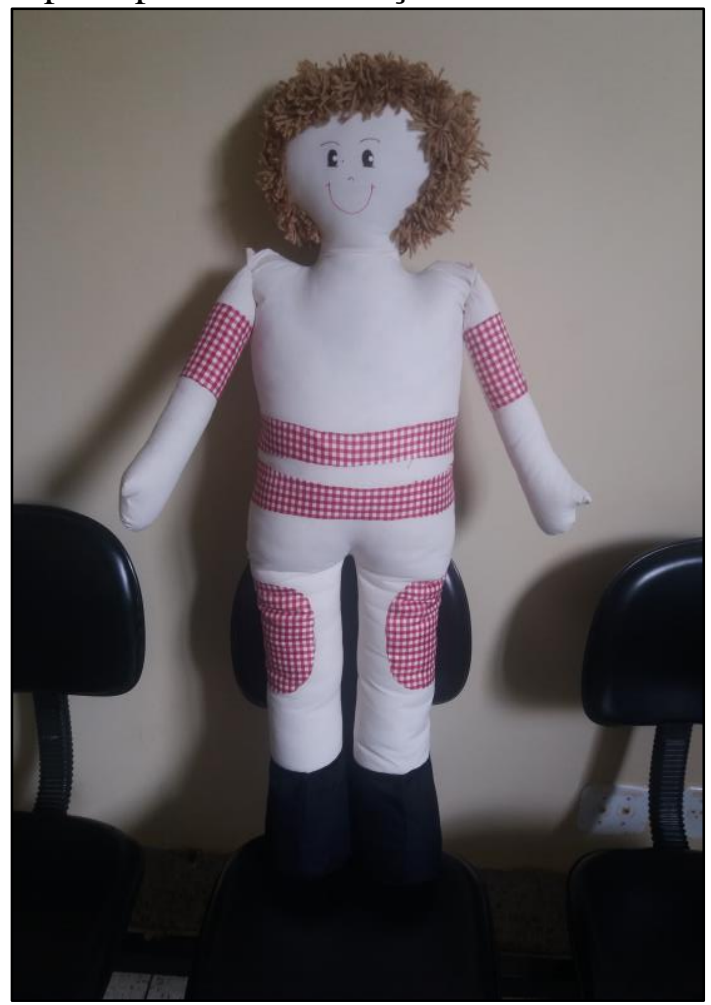

FONTE: Os autores (2018)

d) Cartilha instrucional desenvolvida com orientações básicas sobre diabetes e insulinoterapia, evidenciando aspectos indispensáveis sobre a técnica de administração subcutânea, rodízio de aplicações e monitorização glicêmica, com espaços para anotações importantes, como valores glicêmicos; e

e) Insumos utilizados na auto-aplicação para um momento prático, tais como agulhas e seringas próprias para administração subcutânea, aparelho de monitorização dos níveis glicêmicos (glicosímetro).

\section{RESULTADOS E DISCUSSÕES}

Estratégias de educação em saúde individual e em grupo: relato das experiências Ações de cuidado educativo em grupo 
Os grupos eram formados por 5 usuários, em média, que participavam das atividades propostas pela equipe do projeto durante uma hora e meia. Em todos os encontros, os acadêmicos de Enfermagem conduziam os processos, supervisionados por uma enfermeiradocente responsável pelo projeto. Para iniciar as atividades durante os encontros, optou-se pela utilização de dinâmicas de acolhimento e apresentação, seguidos de uma conversa informal para o estímulo ao envolvimento e participação dos usuários.

O método de abordagem escolhido para atuar junto aos usuários participantes foi baseado na utilização de tecnologias leve-duras em saúde, voltadas para a promoção do autocuidado e protagonismo dos usuários no que diz respeito à sua própria saúde, oportunizando um espaço dialógico de compartilhamento de dúvidas e questionamentos sobre a autoadministração de insulina subcutânea, a troca de experiências sobre esta modalidade de tratamento e a exposição dos principais obstáculos encontrados para o tratamento insulínico.

As atividades tiveram como moldes os preceitos da metodologia ativa, a qual considera como viáveis novas formas de ensino-aprendizagem e de organização curricular na perspectiva de integrar teoria-prática, ensino-serviço, as disciplinas e as diferentes profissões da área da saúde (MITRE et al., 2008), além de buscar desenvolver a capacidade de reflexão sobre problemas reais e a formulação de ações originais e criativas capazes de transformar a realidade social.

A questão preponderante para a escolha do método de abordagem aos usuários foi a possibilidade de fuga do padrão tradicional com o qual a educação em saúde é trabalhada na atenção primária. Durante o contato com os usuários, procurou-se utilizar de uma linguagem de fácil compreensão, clara e objetiva, com palavras que faziam parte do cotidiano dos mesmos. Os acadêmicos, nesse momento, assumiram um papel de direcionadores e facilitadores das temáticas abordadas nas ações, intervindo com esclarecimentos e orientações, caso fossem necessários.

Os principais direcionamentos voltaram-se para o entendimento facilitado sobre a doença, considerações a respeito da autoadministração da insulina e monitorização dos níveis glicêmicos.

\section{Consultas de enfermagem individuais}

As consultas individuais foram realizadas com usuários que iniciaram o tratamento com insulina durante a vigência do projeto e com indivíduos triados a partir das orientações feitas em grupo, aqueles em que se percebeu a necessidade de uma atenção individualizada. Cada 
consulta tinha duração média de uma hora, durante as quais foram aferidos sinais vitais, medidas antropométricas e glicemia capilar.

A consulta direcionou-se em enfatizar pontos principais relacionados à doença, contemplando dieta e prática de exercícios físicos; conceito, importância de conhecer todas as áreas de aplicação, melhor forma de desprezar o que foi utilizado, modos de administração e possíveis efeitos adversos do uso da insulina; bem como a verificação diária de níveis de glicemia.

De início foi realizada uma conversa de caráter informal entre os participantes do projeto e usuários, para fins de conhecimento e posterior adequação de orientações, partindo do que estes já conheciam e abordando o que precisava ser apresentado e/ou reiterado. Para este fim os extensionistas atentaram previamente para os determinantes socioeconômicos, culturais e clínicos dos usuários, o que impulsionou a utilização de linguagem acessível buscando integral entendimento do que estava sendo explicitado.

O familiar, que é quem convive com o usuário e pode supervisioná-lo no tratamento, também foi estimulado a participar de consultas médicas e de enfermagem, recebendo orientações sobre os cuidados a serem tomados, pois a maioria dos usuários configuravam-se em pessoas idosas, que apresentam muitas vezes dificuldades em seguir corretamente as instruções e dar seguimento adequado ao tratamento e prevenção de agravos.

O momento da prática foi posterior a esse primeiro momento, permitindo aos participantes terem contato com os materiais utilizados, assim como capacitação quanto à técnica de preparo, administração e verificação glicêmica. Houve ênfase no cálculo de dose correta em unidades internacionais em seringa própria de insulina, pois este foi um grande motivo de dúvida, o que poderia levar ao cálculo e administração de quantidade errada de insulina, comprometendo o tratamento, e finalmente as múltiplas regiões anatômicas utilizadas na administração de insulina foram identificadas através do boneco de tecido feito para esta finalidade. A prática destes procedimentos foi oportunizada para os usuários e, se houvesse dúvidas, os extensionistas estavam presentes para respondê-las.

\section{CONCLUSÃO}

A realidade a qual os usuários estavam inseridos pôde ser conhecida pelos discentes, em especial no que se refere às dificuldades frente à terapêutica. A ação proposta foi recebida de forma satisfatória pelos usuários, sendo essa receptividade percebida pela forma em que o 
método foi acolhido, com total interesse do público-alvo pela temática em questão, tão próxima da realidade diária dos mesmos.

A participação efetiva dos usuários foi notória, compartilhando saberes, sanando dúvidas e curiosidades no decorrer da conversa, expondo suas experiências subjetivas. A possibilidade de intervir na realidade com o uso da educação em saúde representou um grande aprendizado não contemplado pelo currículo da graduação.

A enfermagem em seu caráter assistencial também foi evidenciada, dando destaque ao autocuidado, trabalhando para reduzir abandonos ao tratamento, beneficiando a qualidade de vida do usuário, bem como a família, sociedade e sistema público.

Percebeu-se com a vivência a essencialidade da educação terapêutica na atenção primária para informar, motivar e fortalecer a pessoa e a família, cabendo aos profissionais de saúde, especialmente os enfermeiros, a adoção de atividades educativas em grupo para enfatizarem programas de atenção integral, sendo possível por meio destas construir alternativas, transformar comportamentos desfavoráveis à saúde e apoiar o fortalecimento de atitudes saudáveis, no intuito de possibilitar um engajamento verdadeiro quanto ao tratamento com insulina, controle de índices glicêmicos e diminuição das complicações advindas do diabetes mellitus.

\section{REFERÊNCIAS}

BARROS, A. E.; SOUZA, E. N. Autoaplicação de insulina: atitudes de um grupo de diabéticos. Revista de Enfermagem UFPE On Line, v. 5, n. 3, p. 593-603, maio 2011. Disponível em: https://periodicos.ufpe.br/revistas/revistaenfermagem/article/download/6764/6011. Acesso em: 31 de março de 2018.

CONSELHO FEDERAL DE ENFERMAGEM (COFEN). Resolução no 358, de 15 de outubro de 2009. Dispõe sobre a Sistematização da Assistência de Enfermagem e a implementação do Processo de Enfermagem em ambientes, públicos ou privados, em que ocorre o cuidado profissional de Enfermagem, e dá outras providências. COFEN, 2009. Disponível em: http://www.cofen.gov.br/resoluo-cofen-3582009_4384.html. Acesso em: 30 de março de 2018.

COSTA, D. V. P. Empoderamento na educação em grupo de diabetes na atenção primária à saúde. Dissertação (Mestrado em Enfermagem), 108 p. Programa de PósGraduação da Escola de Enfermagem da Universidade Federal de Minas Gerais (EEUFMG), Belo Horizonte, Minas Gerais. 
CYRINO, A. P.; SCHRAIBER, L. B; TEIXEIRA, R. R. Education for type 2 diabetes mellitus self-care: from compliance to empowerment. Interface (Botucatu), Botucatu, v. 13, n. 30, Sept. 2009. Disponível em: www.scielo.br/pdf/icse/v13n30/v13n30a09.pdf. Acesso em: 1 de abril de 2018.

DIAS, V. P.; SILVEIRA, D. T.; WITT, R. R. Educação em saúde: o trabalho de grupos em atenção primária. Revista APS, v. 12, n. 2, p. 221-227, abr./jun. 2009.

GUIDONI, C. M. et al. Assistência ao diabetes no Sistema Único de Saúde: análise do modelo atual. Brazilian Journal of Pharmaceutical Sciences, v. 45, n. 1., jan./mar., 2009. Disponível em: http://www.scielo.br/pdf/bjps/v45n1/05.pdf. Acesso em: 31 de março de 2018.

LEI N ${ }^{\circ}$ 11.347 DE 27 DE SETEMBRO DE 2006. Dispõe sobre a distribuição gratuita de medicamentos e materiais necessários à sua aplicação e à monitorização de glicemia capilar aos portadores de diabetes inscritos em programas de educação para diabéticos. Diário Oficial da União (Brasília), 2006.

MENEZES, K. F. P; AVELINO, P. R. Grupos operativos na Atenção Primária à Saúde como prática de discussão e educação: uma revisão. Cadernos de Saúde Coletiva, Rio de Janeiro, v. 24, n. 1, p. 124-130, 2016.

MITRE, S. M. et al. Metodologias ativas de ensino-aprendizagem na formação profissional em saúde: debates atuais. Ciência \& Saúde Coletiva, Rio de Janeiro, v. 13, supl. 2, p. 2133 2144, dez. 2008. Disponível em: http://www.scielo.br/pdf/csc/v13s2/v13s2a18.pdf. Acesso em: 31 de março de 2018.

SOCIEDADE BRASILEIRA DE ENDOCRINOLOGIA E METABOLOGIA (SBEM); ASSOCIAÇÃO BRASILEIRA DE NUTROLOGIA (ABRAN). Diabetes Mellitus Tipo 2: Insulinização. Projeto Diretrizes. Rio de Janeiro: Associação Médica Brasileira e Conselho Federal de Medicina, 2011.

STACCIARINI, T. S. G.; PACE, A. E.; HAAS, V. J. Técnica de autoaplicação de insulina com seringas descartáveis entre os usuários com diabetes mellitus, acompanhados pela Estratégia Saúde da Família. Revista Latino-americana de Enfermagem, v. 17, n. 4, jul./ago., 2009. Disponível em: https://www.revistas.usp.br/rlae/article/view/4027/0. Acesso em: 31 de março de 2018. 